\title{
Quantum and classical separability of spin-orbit laser modes
}

\author{
L. J. Pereira, A. Z. Khoury and K. Dechoum \\ Instituto de Física, Universidade Federal Fluminense, 24210-346 Niterói - RJ, Brasil.
}

\begin{abstract}
In this work we investigate the quantum noise properties of polarization vortices in connection with an intensity based Clauser-Horne-Shimony-Holt inequality for their spin-orbit separability. We evaluate the inequality for different input quantum states and the corresponding intensity fluctuations. The roles played by coherence and photon number squeezing provide a suitable framework for characterizing pure state spin-orbit entanglement. Structural inseparability of the spin-orbit mode requires coherence, an issue concerning either classical or quantum descriptions. In both cases, it can be witnessed by violation of this intensity based CHSH inequality. However, in the quantum domain, entanglement requires both coherence and reduced photon number fluctuations.
\end{abstract}

PACS numbers: 03.65.Ud, 03.67.Mn, 42.50.Dv

\section{INTRODUCTION}

As a major resource for quantum information processing, entangled states have been intensively studied in a wide variety of quantum systems. From the mathematical point of view, when pure states are considered, they correspond to state vectors of a combined system that cannot be factorized as a tensor product of vectors belonging to the Hilbert spaces of the individual subsystems. In the general case, mixed entangled states cannot be written as a convex combination of projectors on factorized vectors. From the physical point of view, these states exhibit strong correlations when the individual systems are locally measured in different (non orthogonal) bases, as in the well known setting for the Clauser-HornShimony-Holt (CHSH) inequality [1]. For example, in photonic systems it has been tested with polarization entangled photon pairs [2]. One can also encode a pair of qubits on the spin and orbital angular momentum of a single photon [3]. Combination of the two degrees of freedom leads to interesting applications on quantum information protocols including polarization controlled quantum imaging [4, 5], algorithms [6], gates [7-11], cryptography 12 14], teleportation [15 18], and topological phases [19, 20]. Of course, the CHSH inequality can be naturally applied to the spin-orbit degrees of freedom as well [21 23].

Despite being frequently addressed in the quantum domain, tensor product structures are also present in the classical description of the electromagnetic field. We may quote the aforementioned example of the spin and orbital angular momentum carried by the optical field. While spin is related to the polarization, orbital angular momentum (OAM) has its origin in the wavefront structure [24]. In a paraxial beam, these two degrees of freedom can be addressed independently and a combined mode basis can be built from a tensor product. Of course, one can also conceive non-separable spin-orbit modes and build a bridge for an interesting analogy with entangled states in quantum mechanics. Many concepts can be borrowed from quantum information theory, with a special role for the $\mathrm{CHSH}$ inequality as a non separability witness. This classical analogy of the $\mathrm{CHSH}$ inequality for optical degrees of freedom was suggested in Ref. 25]. The spin-orbit version of the experiment was published in Ref. 22] together with a theoretical description, including a brief quantum optical approach. Since then, other experimental [26, 27] and theoretical [28 31] works have addressed this issue. Spin-orbit entanglement has also been discussed in the quantum optical domain. The connection between classical inseparability and quantum entanglement was addressed in Ref. 32], where the fundamental properties of cylindrically polarized modes were thoroughly investigated. Experimental tools for squeezing and entangling spin and orbital degrees of freedom were developed in Refs. 33, 34].

In this work, we discuss quantum optical aspects of the spin-orbit separability, with a special attention to the roles played by coherence and sub Poissonian photon number distribution [35-37] in connection with the spin-orbit Bell measurements performed in Ref. 22]. We will consider different input states, covering the main attributes directly related to the spin-orbit separability, especially coherence and quantum entanglement. The manuscript is organized as follows: in section [1] we review the basic description of polarization vortices as nonseparable spin-orbit modes. These modes are then quantized in section III where different mode partitions are envisaged. The measurement scheme on which we base our theory is described in section IV] and the corresponding average intensities and quantum noise are derived in section $\nabla$ for different input states. Finally, we summarize our conclusions in section [VI]

\section{POLARIZATION VORTICES AS NON-SEPARABLE SPIN-ORBIT MODES}

In this section we discuss the notion of mode entanglement and its subtle distinction from quantum state entanglement. Both rely on the concept of non separability in a tensor product vector space. However, the vector structure of electromagnetic modes is not exclusive to quantum theory, it already occurs in classical electrodynamics. The polarization of a light beam is directly re- 
lated to the vector nature of the electromagnetic field. At the same time, a vector space structure can be assigned to the spatial functions used to describe a paraxial beam. Therefore, the complete mode structure requires a combination of these two degrees of freedom in the form of a tensor product between the two vector spaces. For example, we can combine linear polarization vectors with Hermite-Gaussian spatial functions, which are discrete solutions of the paraxial wave equation in rectangular coordinates.

We shall label the linear polarization directions with capital letters $H$ and $V$, associated with the unit vectors $\hat{\mathbf{e}}_{H} \equiv \hat{\mathbf{x}}$ and $\hat{\mathbf{e}}_{V} \equiv \hat{\mathbf{y}}$. The first-order Hermite-Gaussian modes oriented along the horizontal and vertical directions will be labeled with small case letters $h$ and $v$, respectively, associated with the spatial functions

$$
\begin{aligned}
& \psi_{h}(x, y, z)=\mathcal{N} x \exp \left[-\frac{\left(x^{2}+y^{2}\right)}{2 w^{2}(z)}+i \phi(x, y, z)\right], \\
& \psi_{v}(x, y, z)=\mathcal{N} y \exp \left[-\frac{\left(x^{2}+y^{2}\right)}{2 w^{2}(z)}+i \phi(x, y, z)\right]
\end{aligned}
$$

where $\mathcal{N}$ is a normalization constant and $\phi(x, y, z)$ is the phase distribution. The most general first-order vector mode can be written as

$$
\begin{aligned}
\Psi(\mathbf{r}) & =A_{H h} \psi_{h} \hat{\mathbf{e}}_{H}+A_{H v} \psi_{v} \hat{\mathbf{e}}_{H}+A_{V h} \psi_{h} \hat{\mathbf{e}}_{V} \\
& +A_{V v} \psi_{v} \hat{\mathbf{e}}_{V}
\end{aligned}
$$

where $\sum_{\mu \nu}\left|A_{\mu \nu}\right|^{2}=1$. We can borrow the definition of concurrence from quantum information theory to characterize the spin-orbit separability

$$
\mathcal{C}=2\left\|A_{H h} A_{V v}-A_{H v} A_{V h}\right\|,
$$

so that $\mathcal{C}=0$ for product modes and $0<\mathcal{C} \leq 1$ for non factorisable ones.

A first-order spin-orbit mode basis can also be built with maximally non-separable modes analogous to Bell states in quantum mechanics

$$
\begin{aligned}
& \boldsymbol{\Psi}_{ \pm}(\mathbf{r})=\frac{\psi_{h} \hat{\mathbf{e}}_{H} \pm \psi_{v} \hat{\mathbf{e}}_{V}}{\sqrt{2}} \\
& \boldsymbol{\Phi}_{ \pm}(\mathbf{r})=\frac{\psi_{h} \hat{\mathbf{e}}_{V} \pm \psi_{v} \hat{\mathbf{e}}_{H}}{\sqrt{2}} .
\end{aligned}
$$

They correspond to non uniform polarizations on the wavefront. Two simple examples of maximally non separable modes are the well known radially $\left(\boldsymbol{\Psi}_{+}\right)$and azimuthally $\left(\boldsymbol{\Phi}_{-}\right)$polarized modes, characterized by the polarization patterns shown in Fig.(11). Numerous methods for their production are available in the literature. They have attracted a considerable attention for their special focusing properties. It is easy to check from Eq.(3) that $\mathcal{C}=1$ for all elements of the basis given by Eq.(4).

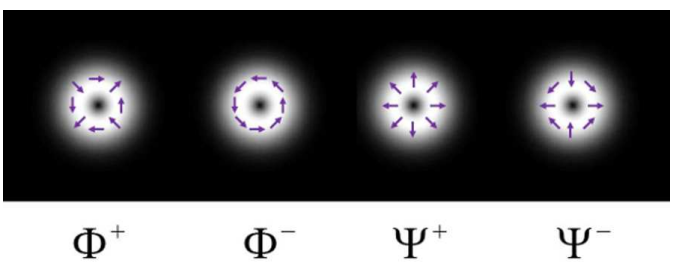

FIG. 1: First order polarization vortices analogous to the four Bell states.

\section{THE QUANTIZED SPIN-ORBIT MODES}

Let us consider the Heisenberg operator describing the positive frequency component of the electric field associated with a first-order paraxial beam propagating along $z$. In the separable mode basis $\left\{\psi_{\nu}(\mathbf{r}) \hat{\mathbf{e}}_{\mu}\right\}$, with $\mu=H, V$ and $\nu=h, v$, it reads

$$
\mathbf{A}^{(+)}(\mathbf{r}, t)=e^{i(k z-\omega t)} \sum_{\mu, \nu} a_{\mu \nu} \psi_{\nu}(\mathbf{r}) \hat{\mathbf{e}}_{\mu}
$$

where $a_{\mu \nu}$ is the operator that annihilates a photon on mode $\psi_{\nu}(\mathbf{r}) \hat{\mathbf{e}}_{\mu}$. As usual, we can also define new annihilation operators when a different mode basis is used to decompose the field operator. For the Bell modes defined in section [II, we obtain the following transformation equations for annihilation operators

$$
\begin{aligned}
& a_{\Psi_{ \pm}}=\frac{a_{H h} \pm a_{V v}}{\sqrt{2}}, \\
& a_{\Phi_{ \pm}}=\frac{a_{H v} \pm a_{V h}}{\sqrt{2}},
\end{aligned}
$$

and the corresponding conjugate transformations for the creation operators. The electric field operator can then be written as

$$
\mathbf{A}^{(+)}(\mathbf{r}, t)=e^{i(k z-\omega t)} \sum_{ \pm} a_{\Psi_{ \pm}} \boldsymbol{\Psi}_{ \pm}+a_{\Phi_{ \pm}} \mathbf{\Phi}_{ \pm} .
$$

Now it is interesting to investigate the transformations for different kinds of states. A Fock basis of the Hilbert space can be built for either mode decompositions by acting on the vacuum state with the corresponding creation operators. Let us consider, for example, an $N$-photon Fock state on mode $\boldsymbol{\Psi}_{+}$, with all other modes empty. For simplicity, we shall omit the empty modes in the state vector, unless they are directly involved in the transformations that we are interested, however we must keep in mind that we will be always dealing with a multimode Hilbert space. Thus, for the mode in question we have

$$
|N\rangle_{\Psi_{+}}|0\rangle_{\Psi_{-}}=\frac{\left(a_{\Psi_{+}}^{\dagger}\right)^{N}}{\sqrt{N !}}|v a c\rangle .
$$

If we use the conjugate transformations (6) and write the creation operator for $\boldsymbol{\Psi}_{+}$in terms of those for $H h$ and 
$V v$, we obtain

$$
|N\rangle_{\Psi_{+}}|0\rangle_{\Psi_{-}}=\sum_{n=0}^{N} \frac{\sqrt{N !}\left(a_{H h}^{\dagger}\right)^{n}\left(a_{V v}^{\dagger}\right)^{N-n}}{2^{N / 2} n !(N-n) !}|v a c\rangle .
$$

From the analogous definitions of the Fock states on modes $H h$ and $V v$, we readily identify them in the right hand side of Eq.(9), so that

$$
|N\rangle_{\Psi_{+}}|0\rangle_{\Psi_{-}}=\sum_{n=0}^{N} \sqrt{\frac{N ! / 2^{N}}{n !(N-n) !}}|n\rangle_{H h}|N-n\rangle_{V v} .
$$

For example, a single photon Fock state on mode $\boldsymbol{\Psi}_{+}$ can be written

$$
|1\rangle_{\Psi_{+}}|0\rangle_{\Psi_{-}}=\frac{|1\rangle_{H h}|0\rangle_{V v}+|0\rangle_{H h}|1\rangle_{V v}}{\sqrt{2}} .
$$

Note that the left hand side of Eqs. (10) and (11) has a product state while the right hand side has an entangled one. Of course, entanglement is independent of the basis chosen for the Hilbert space, however these equations are not a change of basis, they correspond to different mode partitions of the electromagnetic field. Therefore, the mode basis must not be confused with the quantum state basis. In this sense, we prefer to employ the term mode partition in order to avoid this kind of confusion.

Entanglement does depend on the way the subsystems are defined and, although this partition is usually evident for material systems, it is not so clear for the electromagnetic field since one often has more than one mode decomposition adapted to the same boundary conditions. Therefore, we must be careful when comparing entanglement in the two cases. In this respect, pure coherent states play a very special role because they are always a product state in whatever mode decomposition. Indeed, let

$$
D_{\Psi_{+}}(u)=\exp \left(u a_{\Psi_{+}}^{\dagger}-u^{*} a_{\Psi_{+}}\right)
$$

be the displacement operator that produces the coherent state $|u\rangle_{\Psi_{+}}|0\rangle_{\Psi_{-}}$when acting on the vacuum state. Since $a_{H h}$ and $a_{V v}$ commute, it can be factorized as a product between the corresponding displacement operators of modes $H h$ and $V v$. From Eqs.(6) one easily obtains

$$
D_{\Psi_{+}}(u)=D_{H h}(u / \sqrt{2}) D_{V v}(u / \sqrt{2}),
$$

so that the coherent states in the two mode decompositions are related by

$$
|u\rangle_{\Psi_{+}}|0\rangle_{\Psi_{-}}=|u / \sqrt{2}\rangle_{H h}|u / \sqrt{2}\rangle_{V v},
$$

which is a product state in any case. From this discussion we develop some intuition about the role played by photon number noise in the interplay between mode separability and quantum entanglement. We next discuss this relationship more closely in the context of the experiment done in Ref.[22].

\section{SPIN-ORBIT BELL MEASUREMENTS}

Let us consider the sketch shown in Fig.(2) for a spinorbit Bell measurement as the one done in [22]. An input mode initially prepared in some quantum state $\left|\varphi_{0}\right\rangle$ enters a Mach-Zehnder interferometer with an extra mirror in one arm. This type of interferometer will be designated by the acronym MZIM [38]. When the two arms are equilibrated, the interferometer sorts even and odd modes at different output ports, the components $H h$ and $V v$ exit from the even port while $H v$ and $V h$ exit from the odd port. The input beams are described by the electric field operators

$$
\mathbf{A}_{j}^{(+)}(\mathbf{r}, t)=e^{i\left(\mathbf{k}_{j} \cdot \mathbf{r}-\omega t\right)} \sum_{\mu, \nu} a_{\mu \nu}^{j} \psi_{\nu}(\mathbf{r}) \hat{\mathbf{e}}_{\mu}
$$

where $\mu=H, V$ and $\nu=h, v$ refer to polarization and transverse mode orientation respectively, $j=1,2$ refers to the input port, and $a_{\mu \nu}^{j}$ is the corresponding annihilation operator. The input field coming through port 1 will be transmitted through unitary transformation elements acting on the transverse mode and polarization degrees of freedom. These elements are used to set the bases of the Bell measurement. They can be a Dove prism (DP) for transverse mode and a half wave plate (HWP) for polarization transformation. When the HWP is oriented at an angle $\alpha / 2$ and the DP at $\beta / 2$, the annihilation operators are transformed according to

$$
a_{\mu \nu}^{\prime 1}(\alpha, \beta)=\sum_{\epsilon \delta} U_{\mu \nu}^{\epsilon \delta} a_{\epsilon \delta}^{1},
$$

where $U$ is the unitary transformation describing the combined action of the two elements. The HWP and the DP both act in a similar manner, they produce a reflection operation along their orientation axis. Therefore, their combined action can be written as the tensor product $U=T(\alpha) \otimes T(\beta)$, where

$$
T(\theta)=\left(\begin{array}{cc}
\cos \theta & \sin \theta \\
\sin \theta & -\cos \theta
\end{array}\right) .
$$

In matrix notation we have

$$
\left(\begin{array}{l}
a_{H h}^{\prime 1} \\
a_{H v}^{\prime 1} \\
a_{V h}^{\prime 1} \\
a_{V v}^{\prime 1}
\end{array}\right)=\left(\begin{array}{cc}
\cos \alpha & \sin \alpha \\
\sin \alpha & -\cos \alpha
\end{array}\right) \otimes\left(\begin{array}{cc}
\cos \beta & \sin \beta \\
\sin \beta & -\cos \beta
\end{array}\right)\left(\begin{array}{l}
a_{H h}^{1} \\
a_{H v}^{1} \\
a_{V h}^{1} \\
a_{V v}^{1}
\end{array}\right) .
$$

The same decomposition also applies to the output field operators

$$
\mathbf{B}_{j}^{(+)}(\mathbf{r}, t)=e^{i\left(\mathbf{k}_{j} \cdot \mathbf{r}-\omega t\right)} \sum_{\mu, \nu} b_{\mu \nu}^{j} \psi_{\nu}(\mathbf{r}) \hat{\mathbf{e}}_{\mu},
$$

where $b_{\mu \nu}^{j}$ is the annihilation operator corresponding to mode $\mu \nu$ at output $j$.

One of the output ports of the interferometer will combine the odd modes from the vacuum input with the even 


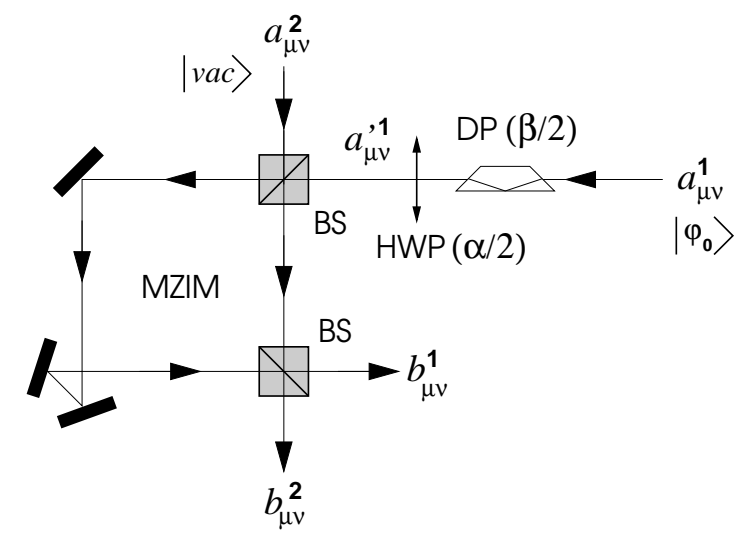

FIG. 2: Scheme for the spin-orbit Bell measurement.

modes from the excited input and the opposite combination is performed in the other output port. The quantized mode amplitudes $b_{\mu \nu}$ are then determined by the input-output relations for the interferometer

$$
\begin{array}{ll}
b_{H h}^{1}=a_{H h}^{\prime 1}, & b_{H h}^{2}=a_{H h}^{2}, \\
b_{H v}^{1}=a_{H v}^{2}, & b_{H v}^{2}=a_{H v}^{\prime 1}, \\
b_{V h}^{1}=a_{V h}^{2}, & b_{V h}^{2}=a_{V h}^{\prime 1}, \\
b_{V v}^{1}=a_{V v}^{\prime 1}, & b_{V v}^{2}=a_{V v}^{2} .
\end{array}
$$

We now define the intensity operators integrated over the detectors area

$$
\hat{I}_{j}=\int_{D_{j}} \mathbf{B}_{j}^{(-)} \cdot \mathbf{B}_{j}^{(+)} d^{2} \mathbf{r} .
$$

From the input-output relations of the measurement apparatus (transformation elements plus interferometer) one finds

$$
\begin{aligned}
& \hat{I}_{1}=\hat{I}_{H h}^{1}+\hat{I}_{V v}^{1}+\hat{I}_{H v}^{1}+\hat{I}_{V h}^{1}, \\
& \hat{I}_{2}=\hat{I}_{H v}^{2}+\hat{I}_{V h}^{2}+\hat{I}_{H h}^{2}+\hat{I}_{V v}^{2},
\end{aligned}
$$

where $\hat{I}_{\mu \nu}^{j}=b_{\mu \nu}^{j \dagger} b_{\mu \nu}^{j}$. Note that modes $H v$ and $V h$ on output port 1 are not excited, as well as modes $H h$ and $V v$ on output port 2 . Therefore, they do not contribute to intensity measurements and the relevant operators at the output of the interferometer are

$$
\begin{aligned}
I_{t o t} & =\hat{I}_{1}+\hat{I}_{2}, \\
M(\alpha, \beta) & =\hat{I}_{1}-\hat{I}_{2} .
\end{aligned}
$$

The total input-output intensity does not depend on the measurement settings $(\alpha, \beta)$.

In analogy with the parameter used in the ClauserHorn-Shimony-Holt (CHSH) inequality, we define

$$
S=\frac{\left\langle M(\alpha, \beta)+M\left(\alpha, \beta^{\prime}\right)-M\left(\alpha^{\prime}, \beta\right)+M\left(\alpha^{\prime}, \beta^{\prime}\right)\right\rangle}{\left\langle I_{t o t}\right\rangle}
$$

where the averages are calculated with the initial quantum state $\left|\varphi_{0}\right\rangle$. For single photon states, the normalized detected intensities correspond to detection probabilities and one finds the usual scenario for Bell measurement where $2<S<2 \sqrt{2}$ for single photon spin-orbit entangled states. As discussed in Ref. [22], this violation can be achieved with a polarization vortice coherent state, which is a product state in the $H h$ and $V v$ decomposition, as shown in Eq.(14). Therefore, in order to evidence quantum behavior, it will be necessary to monitor the intensity difference noise $\Delta M^{2}(\alpha, \beta)=$ $\left\langle M^{2}(\alpha, \beta)\right\rangle-\langle M(\alpha, \beta)\rangle^{2}$ and compare it with the corresponding shot noise limit. This figure of merit will be used in the next section to set up the comparison between different input states.

\section{INTENSITY AVERAGES AND QUANTUM NOISE}

Let us assume that only modes $a_{H h}^{1}$ and $a_{V v}^{1}$ are initially occupied with some quantum state $\left|\varphi_{0}\right\rangle$, while all other modes are empty. We will proceed as follows, first all output intensity operators are calculated in terms of the input modes (before HWP and DP) using the inputoutput relations outlined above. Then the mean values giving the average intensity and the corresponding quantum fluctuation are calculated with the specified initial state. We will discuss different input states, their similarities and differences.

\section{A. Entangled Fock states}

Let us first consider the single photon entangled state given by Eq.(11). In this case, we have

$$
\frac{\langle M(\alpha, \beta)\rangle}{\left\langle I_{t o t}\right\rangle}=\cos [2(\beta-\alpha)],
$$

where $\left\langle I_{t o t}\right\rangle=1$. For the measurement settings $\alpha=$ $\pi / 8, \alpha^{\prime}=3 \pi / 8, \beta=0, \beta^{\prime}=\pi / 4$, we obtain the limiting value $S=2 \sqrt{2}$ expected for a maximally spin-orbit entangled state. We also evaluate the corresponding intensity difference fluctuations for any setting $(\alpha, \beta)$

$$
\frac{\Delta M^{2}(\alpha, \beta)}{\left\langle I_{t o t}\right\rangle}=\sin ^{2}[2(\beta-\alpha)] .
$$

These results can be easily generalized for the arbitrary $N$-photon entangled state $|N\rangle_{\Psi_{+}}|0\rangle_{\Psi_{-}}$given by Eq.(10) for which $\left\langle I_{t o t}\right\rangle=N$. For the Bell measurement settings we obtain $50 \%$ intensity squeezing. When $\beta-\alpha=m \pi / 2(m \in \mathbb{Z})$, all photons exit through the same output port and the intensity difference is perfectly squeezed.

Note that inter-mode coherence is a key ingredient for violation of the CHSH inequality, since the summations on the right hand side of Eqs. (10) and (11) involve a 
coherent superposition with well defined relative phases. When those are randomized, one gets statistical mixtures of Fock states which no longer violate the inequality, as we will see next.

\section{B. Mixed Fock states}

It will be interesting to evaluate the spin-orbit $\mathrm{CHSH}$ inequality for a mixed Fock state exhibiting strong photon number correlations but no entanglement. This state can be represented by the density matrix

$\rho_{N}=\sum_{n=0}^{N} \frac{N ! / 2^{N}}{n !(N-n) !}|n\rangle\left\langle\left. n\right|_{H h} \otimes \mid N-n\right\rangle\left\langle N-\left.n\right|_{V v}\right.$,

with a well defined total photon number $N$, randomly distributed between modes $H h$ and $V v$. For this state we obtain

$$
\frac{\langle M(\alpha, \beta)\rangle}{\left\langle I_{t o t}\right\rangle}=\cos 2 \alpha \cos 2 \beta
$$

what results in $S=\sqrt{2}$ for the measurement settings.

The intensity difference noise is

$$
\begin{aligned}
\frac{\Delta M^{2}(\alpha, \beta)}{\left\langle I_{t o t}\right\rangle} & =\sin ^{2} 2 \alpha+\sin ^{2} 2 \beta \\
& +\left(\frac{\left\langle I_{t o t}\right\rangle-3}{2}\right) \sin ^{2} 2 \alpha \sin ^{2} 2 \beta,
\end{aligned}
$$

which can exhibit perfect squeezing for specific settings $(\alpha, \beta)$, but in general scales with the photon number. For example, if we set $\alpha=\beta=\pi / 4$, expression (29) gives $(N+1) / 2$ while (26) predicts perfect squeezing.

\section{Photon number Werner states}

In order to capture the role played by entanglement in the quantum noise properties of Fock states, let us consider a partially entangled state of the kind

$$
\rho_{N}(p)=p|N\rangle\left\langle\left. N\right|_{\Psi_{+}} \otimes \mid 0\right\rangle\left\langle\left. 0\right|_{\Psi_{-}}+(1-p) \rho_{N},\right.
$$

where $\rho_{N}$ is given by Eq.(27). It is analogous to the Werner states frequently used in quantum information science to discuss correlations present in partially entangled states [39]. The average intensity difference for setting $(\alpha, \beta)$ is

$\frac{\langle M(\alpha, \beta)\rangle}{\left\langle I_{\text {tot }}\right\rangle}=p \cos [2(\beta-\alpha)]+(1-p) \cos 2 \alpha \cos 2 \beta$,

which gives $S=(1+p) \sqrt{2}$, corresponding to a violation threshold at $p=\sqrt{2}-1$.
The intensity noise can be readily calculated as a combination of the results given by Eqs.(26) and (29). In fact, one can easily verify the relation

$$
\begin{aligned}
\left\langle\Delta M^{2}\right\rangle_{W} & =p\left\langle\Delta M^{2}\right\rangle_{\text {pure }}+(1-p)\left\langle\Delta M^{2}\right\rangle_{\text {mix }} \\
& +p(1-p)\left[\langle M\rangle_{\text {pure }}-\langle M\rangle_{\text {mix }}\right]^{2}
\end{aligned}
$$

where $\left\langle\Delta M^{2}\right\rangle_{\text {pure }}$ is given by Eq.(26), which scales as $\left\langle I_{\text {tot }}\right\rangle$ while $\left\langle\Delta M^{2}\right\rangle_{\text {mix }}$ is given by Eq.(29), which scales as $\left\langle I_{t o t}\right\rangle^{2}$. Note that both $\langle M\rangle_{\text {pure }}$ and $\langle M\rangle_{\text {mix }}$ are proportional to $\left\langle I_{t o t}\right\rangle$, so that $\left\langle\Delta M^{2}\right\rangle_{W} /\left\langle I_{t o t}\right\rangle$ scales as $\left\langle I_{t o t}\right\rangle$.

\section{Pure coherent state}

For a two-mode coherent state $|u\rangle_{\Psi_{+}}|0\rangle_{\Psi_{-}}$given by Eq. (14) we obtain

$$
\frac{\langle M(\alpha, \beta)\rangle}{\left\langle I_{t o t}\right\rangle}=\cos [2(\beta-\alpha)],
$$

with $\left\langle I_{t o t}\right\rangle=|u|^{2}$, giving the same limiting value $S=$ $2 \sqrt{2}$ as for the entangled Fock state, however, the input state is now factorized. In order to distinguish the two cases, we calculate the intensity difference noise to find

$$
\frac{\Delta M^{2}(\alpha, \beta)}{\left\langle I_{\text {tot }}\right\rangle}=1
$$

so that shot noise is expected for any $(\alpha, \beta)$ settings, which is radically distinguished from the corresponding result for entangled Fock states.

\section{E. Mixed coherent state}

We now investigate the role played by coherence in the violation of the spin-orbit CHSH inequality. Suppose a laser beam is initially prepared in a coherent state $|u\rangle_{H h}|0\rangle_{V v}$. This beam is then split in two equal parts, one of them is transformed to mode $V v$ and sent to a beam splitter where it gets contaminated with an independent laser also prepared in $V v$. Finally, the mixed $V v$ mode is recombined with $H h$ on a polarizing beam splitter (PBS) as sketched in Fig.(3). Since the two lasers have random relative phase, the quantum state describing the final beam can be written as the following statistical mixture

$$
\rho_{0}=|u\rangle\left\langle\left. u\right|_{H h} \otimes \int \frac{d \theta}{2 \pi} \mid u^{\prime}(\theta)\right\rangle\left\langle\left. u^{\prime}(\theta)\right|_{V v},\right.
$$

where $u^{\prime}(\theta)=u\left(r+t e^{i \theta}\right)$, with $r$ and $t$ being the reflection and transmission coefficients of the contaminating coupler. Let $r=\sqrt{R} e^{i \phi}$, where $R$ is the intensity reflectivity and $\phi$ is the phase acquired on reflection, then we obtain

$$
\frac{\langle M(\alpha, \beta)\rangle}{\left\langle I_{\text {tot }}\right\rangle}=\cos 2 \alpha \cos 2 \beta+\sqrt{R} \cos \phi \sin 2 \alpha \sin 2 \beta,
$$


where $\left\langle I_{\text {tot }}\right\rangle=2|u|^{2}$, giving $S=(1+\sqrt{R} \cos \phi) \sqrt{2}$ for the Bell measurement settings. A violation threshold is predicted at $\sqrt{R} \cos \phi=\sqrt{2}-1$. For $R=1$ and $\phi=0$ one recovers the coherent state result given by Eq.(33).

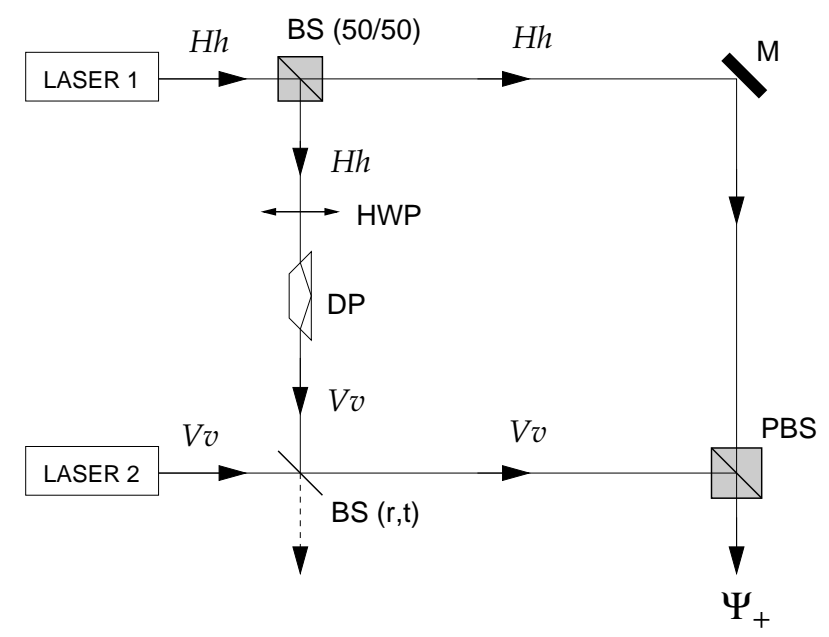

FIG. 3: Proposed scheme to produce an adjustable mixed coherent state.

For a completely incoherent superposition $(R=0)$ we obtain

$$
\frac{\langle M(\alpha, \beta)\rangle}{\left\langle I_{t o t}\right\rangle}=\cos 2 \alpha \cos 2 \beta,
$$

giving $S=\sqrt{2}$ for the Bell measurement settings. This result suggests that coherence is an essential ingredient for violation of the inequality for the intensity average. Meanwhile, the intensity noise for a setting $(\alpha, \beta)$ is

$$
\frac{\Delta M^{2}(\alpha, \beta)}{\left\langle I_{t o t}\right\rangle}=1+\frac{\left\langle I_{t o t}\right\rangle}{2} \sin ^{2} 2 \alpha \sin ^{2} 2 \beta
$$

which scales as $\left\langle I_{t o t}\right\rangle$ and is above shot noise for most settings.

\section{F. Two-mode squeezed vacuum}

It is also interesting to inspect the results obtained for the two-mode squeezed vacuum state, which presents quadrature entanglement. It can be constructed from the action of the two-mode squeezing operator on the vacuum state as given by

$$
\left|\varphi_{s q}\right\rangle=\exp \left(\frac{\zeta^{*} a_{H h} a_{V v}-\zeta a_{H h}^{\dagger} a_{V v}^{\dagger}}{2}\right)|v a c\rangle,
$$

where $\zeta$ is the squeezing parameter and the total intensity is $\left\langle I_{t o t}\right\rangle=2 \sinh ^{2}(|\zeta| / 2)$. In this case we find

$$
\frac{\langle M(\alpha, \beta)\rangle}{\left\langle I_{\text {tot }}\right\rangle}=\cos 2 \alpha \cos 2 \beta,
$$

giving $S=\sqrt{2}$ for the measurement settings, the same result as for the mixed coherent state. For the intensity noise we get

$$
\frac{\Delta M^{2}(\alpha, \beta)}{\left\langle I_{\text {tot }}\right\rangle}=1+\left(\left\langle I_{t o t}\right\rangle+1\right)\left(\frac{1+\cos 4 \alpha \cos 4 \beta}{2}\right),
$$

which also scales as $\left\langle I_{t o t}\right\rangle$ for most settings. Therefore, despite its quadrature entanglement, the quantum properties of squeezed vacuum do not show up in this kind of spin-orbit Bell measurement.

\section{CONCLUSION}

In summary, we have discussed spin-orbit Bell measurements in paraxial polarization vortices from a quantum optical perspective. The vortices are treated as nonseparable modes and their quantum descriptions in different mode decompositions are compared. The mode separability is evaluated with an inequality for the average intensities analogous to the CHSH inequality. It is violated for a single-photon spin-orbit entangled state, as expected, but also by a factorized coherent state. These two limiting cases were experimentally investigated in Refs. 21 23. Therefore, a natural question arises about the interplay between entanglement and coherence in the violation. In this article we compared different input states in order to develop some intuition.

Our results indicate that the roles played by coherence and photon number fluctuations can be summarized by the following examples:

- Without coherence we expect no violation of the intensity based CHSH inequality, as illustrated by the examples with mixed coherent or Fock states (sections $\mathrm{VB}$ and $\mathrm{VE}$ ).

- With coherence and Poissonian distribution for the total photon number, we expect violation but no entanglement, as illustrated by the example with product coherent states (section VD). We remark that pure coherent states are factorized in any mode decomposition but exhibit maximal violation.

- With coherence and reduced (below shot-noise) photon number fluctuations, we expect violation and entanglement, as illustrated by the entangled Fock states (section $\mathrm{VA}$ ).

It is important to stress that in all cases the relevant coherence being considered is the one characterized by inter-mode relative phase, not the absolute one. We also present examples that interpolate between pure and mixed states. Finally, section VFillustrates that quadrature entanglement does not imply in violation of the intensity based CHSH inequality.

Experimentally, structural inseparability and entanglement can be simultaneously certified by measuring 
intensity averages and the corresponding fluctuations. Violation of the CHSH inequality with the averages certify structural inseparability while intensity difference squeezing evidences entanglement. Coherent states should exhibit shot noise level for any measurement settings while photon number squeezed states should present $50 \%$ squeezing for the settings used in the inequality. These results can be experimentally tested with polarization vortices produced on intensity squeezed sources like optical parametric oscillators [40, 41] or pump-noise-supressed diode lasers [42].

\section{Acknowledgments}

Funding was provided by Coordenação de Aperfeiçoamento de Pessoal de Nível Superior (CAPES), Fundação de Amparo à Pesquisa do Estado do Rio de Janeiro (FAPERJ-BR), and Instituto Nacional de Ciência e Tecnologia de Informação Quântica (INCT$\mathrm{CNPq}$ ).
[1] J. F. Clauser, M. A. Horne, A. Shimony, and R. A. Holt, Phys. Rev. Lett. 23, 880 (1969).

[2] A. Aspect, P. Grangier, and G. Roger, Phys. Rev. Lett. 49, 91 (1982).

[3] M. J. Padgett and J. Courtial, Opt. Lett. 24, 430 (1999).

[4] M. França Santos, P. Milman, A. Z. Khoury, and P. H. Souto Ribeiro, Phys. Rev. A 64, 023804 (2001).

[5] D. P. Caetano, P. H. Souto Ribeiro, J. T. C. Pardal, and A. Z. Khoury, Phys. Rev. A 68, 023805 (2003).

[6] A. N. de Oliveira, S. P.Walborn, and C. H. Monken, J. Opt. B: Quantum Semiclassic. Opt. 7, 288 (2005).

[7] L. Marrucci, C. Manzo, and D. Paparo, Phys. Rev. Lett. 96, 163905 (2006).

[8] E. Nagali, F. Sciarrino, F. De Martini, L. Marrucci, B. Piccirillo, E. Karimi, and E. Santamato, Phys. Rev. Lett. 103, 013601 (2009).

[9] E. Nagali, F. Sciarrino, F. De Martini, B. Piccirillo, E. Karimi, L. Marrucci, and E. Santamato, Opt. Express 17, 18745 (2009).

[10] C. E. R. Souza and A. Z. Khoury, Opt. Exp. 18, 92079212 (2010).

[11] A. R. Vieira, M. Hor-Meyll, and A. Z. Khoury, J. Opt. Soc. Am. B 30, 1623 ?1626 (2013).

[12] L. Aolita and S. P. Walborn. Phys. Rev. Lett. 98, 100501 (2007).

[13] C. E. R. Souza, C. V. S. Borges, A. Z. Khoury, J. A. O. Huguenin, L. Aolita, and S. P. Walborn. Phys. Rev. A 77, 032345 (2008).

[14] V. D'Ambrosio, E. Nagali, S. P. Walborn, L. Aolita, S. Slussarenko, L. Marrucci, and F. Sciarrino, Nat. Comm. 3, 961 (2012).

[15] L. Chen and W. She, Phys. Rev. A 80, 063831 (2009).

[16] J. T. Barreiro, T.-C. Wei, and P. G. Kwiat, Phys. Rev. Lett. 105, 030407 (2010).

[17] A. Z. Khoury and P. Milman, Phys. Rev. A 83, 060301(R) (2011).

[18] M. Erhard, H. Qassim, H. Mand, E. Karimi, and R. W. Boyd, arXiv:1404.7573 [quant-ph] (2014).

[19] C. E. R. Souza, J. A. O. Huguenin, P. Milman, and A. Z. Khoury, Phys. Rev. Lett. 99, 160401 (2007).

[20] C. E. R. Souza, J. A. O. Huguenin, and A. Z. Khoury, J. Opt. Soc. Am. A 31, 1007-1012 (2014).

[21] L. Chen and W. She, J. Opt. Soc. Am. B 27, A7-A10 (2010).
[22] C. V. S. Borges, M. Hor-Meyll, J. A. O. Huguenin, and A. Z. Khoury, Phys. Rev. A 82, 033833 (2010).

[23] E. Karimi, J. Leach, S. Slussarenko, B. Piccirillo, L. Marrucci, L. Chen, W. She, S. Franke-Arnold, M. J. Padgett, and E. Santamato, Phys. Rev. A 82, 022115 (2010).

[24] M. Padgett and L. Allen, Contemp. Phys. 41, 275-285 (2000).

[25] R. J. C. Spreeuw, Found. Phys. 28, 361 (1998).

[26] K. H. Kagalwala, G. Di Giuseppe, A. F. Abouraddy, B. E. A. Saleh, Nature Photonics 7, 72 (2013).

[27] A. Vallés, V. DAmbrosio, M. Hendrych, M. Micuda, L. Marrucci, F. Sciarrino, J. P. Torres, arXiv:1407.0144v1 (2014).

[28] B. N. Simon, S. Simon, F. Gori, M. Santarsiero, R. Borghi, N. Mukunda, and R. Simon, Phys. Rev. Lett. 104, 023901 (2010).

[29] X. F. Qian, J. H. Eberly, Opt. Lett. 36, 4110 (2011).

[30] P. Chowdhury, A. S. Majumdar, and G. S. Agarwal, Phys. Rev. A 88, 013830 (2013).

[31] F. De Zela, Phys. Rev. A 89, 013845 (2014).

[32] A. Holleczek, A. Aiello, C. Gabriel, Ch. Marquardt and G. Leuchs, Opt. Exp. 19, 9714 (2011).

[33] C. Gabriel, A. Aiello, W. Zhong, T. G. Euser, N. Y. Joly, P. Banzer, M. Fortsch, D. Elser, U. L. Andersen, Ch. Marquardt, P. St. J. Russell and G. Leuchs Phys. Rev. Lett. 106, 060502 (2011).

[34] C. Gabriel, A. Aiello, S. Berg-Johansen, Ch. Marquardt, G. Leuchs Eur. Phys. J. D 66, 172 (2012).

[35] E. S. Guerra, A. Z. Khoury, L. Davidovich, and N. Zagury, Phys. Rev. A 44, 7785 (1991).

[36] L. Davidovich, S. Y. Zhu, A. Z. Khoury, and C. Su, Phys. Rev. A 46, 1630 (1992).

[37] M. K. Olsen, L. I. Plimak, A. Z. Khoury, Opt. Comm. 201, 373 (2002).

[38] H. Sasada and M. Okamoto, Phys. Rev. A 68, 012323 (2003).

[39] R. F. Werner, Phys. Rev. A 40, 4277 (1989).

[40] A. Heidmann, R. J. Horowicz, S. Reynaud, E. Giacobino, C. Fabre, and G. Camy, Phys. Rev. Lett. 59, 2555 (1987).

[41] B. Coutinho dos Santos, K. Dechoum, and A. Z. Khoury, Phys. Rev. Lett. 103, 230503 (2009).

[42] Y. Yamamoto, S. Machida, and O. Nilsson, Phys. Rev. A 34, 4025 (1986). 